EPJ Web of Conferences 45, 01030 (2013)

DOI: $10.1051 /$ epjconf/20134501030

(c) Owned by the authors, published by EDP Sciences, 2013

\title{
Two-phase distribution in the vertical flow line of a domestic wet central heating system
}

\author{
A.-M.Fsadni ${ }^{1, a}$, Y.T.Ge ${ }^{2}$ \\ ${ }^{1}$ School of Built and Natural Environment, Room: Kirkham 124, University of Central Lancashire, Preston, PR1 2HE, UK \\ ${ }^{2}$ Department of Mechanical Engineering, Room: H118, Brunel University, Uxbridge, UB8 3PH, UK
}

\begin{abstract}
The theoretical and experimental aspects of bubble distribution in bubbly two-phase flow are reviewed in the context of the micro bubbles present in a domestic gas fired wet central heating system. The latter systems are mostly operated through the circulation of heated standard tap water through a closed loop circuit which often results in water supersaturated with dissolved air. This leads to micro bubble nucleation at the primary heat exchanger wall, followed by detachment along the flow. Consequently, a bubbly two-phase flow characterises the flow line of such systems. The two-phase distribution across the vertical and horizontal pipes was measured through a consideration of the volumetric void fraction, quantified through photographic techniques. The bubble distribution in the vertical pipe in down flow conditions was measured to be quasi homogenous across the pipe section with a negligible reduction in the void fraction at close proximity to the pipe wall. Such a reduction was more evident at lower bulk fluid velocities.
\end{abstract}

\section{Introduction}

An emerging trend in the building services industry is the installation of passive deaerators on the flow line of domestic wet central heating systems. To date, no data and theoretical models predicting the two-phase flow characteristics in domestic wet central heating systems are available in the open literature. This gap in literature has prevented essential design improvements to passive deaerators thus impeding the efficiency enhancement of such devices.

Micro bubble formation is a phenomenon affecting a number of industries, including the food, pharmaceutical and chemical industries. Bubble nucleation finds its origins in the presence of superheated or supersaturated solutions. In a domestic central heating system micro bubble formation is the result of water supersaturated with dissolved nitrogen gas, consequently, leading to bubble nucleation on the boiler wall. Such conditions are present during cold start-ups and after the system filling with tap water. Air is mostly absorbed in the system during the cold cycle. At low temperatures, water can absorb the highest quantity of dissolved gasses [1,2]. In most systems this occurs during night time when the system's boiler shuts off.

Data on micro bubble characteristics in central heating systems is important as a good knowledge of

\footnotetext{
a afsadni@uclan.ac.uk
}

the typical bubble characteristics and their distribution is essential for an efficient passive deaeration process. Passive deaerators are more efficient in capturing larger sized bubbles. Deaeration is an important feature of such systems as bubbles accumulate in radiators and result in cold spots, thus reducing the heat transfer area of the radiator and the overall system efficiency. Bubbles are also known to result in unwanted noise, blockages and corrosion. Domestic central heating systems amount to $16 \%$ of the carbon dioxide emissions in the UK [3] and consequently, an optimised system performance should have significant environmental benefits.

A bubbly flow in the heating system flow line results from the detachment of micro bubbles from the boiler wall into the system. Bubbly two-phase flow is characterised by the presence of bubbles with a maximum size much less than the containing vessel or duct. The bubbles are dispersed in a continuous liquid phase [4].

Kashinsky and Randin [5], reported that most studies in vertical two-phase bubbly flow have been done for cocurrent upward flow. Hence, negligble consideration has been given to the downward flow scenario. However, the known studies done in bubbly vertical downward flow by Drew and Lahey [6], Wang et al. [7] Antal et al. [8] and Kashinsky and Randin [5] reported similar void fraction distributions with a quasiconstant void fraction in the core region which drops abruptly to zero as the wall is approached. 
Iskandrani and Kojasoy [9] also reported that, in two-phase vertical bubbly flows, the presence of voids tends to flatten the liquid velocity profile, thus leading to a homogenous phase distribution across the vertical pipe section. More recently, Lu and Tryggvason [10] reported similar trends using direct numerical simulations where the full Navier-Stokes equations were solved by a parallelized front-tracking finite-volume method.

In this paper we will investigate the phenomenon of micro bubbles in wet domestic central heating systems. The typical phase distribution across the vertical pipe section on the boiler flow line will be inves tigated.

\section{Experimental set-up and technique used}

A schematic diagram of the experimental set up is shown in figure 1. The test rig consists of a Vaillaint eco TEC pro 24 condensing boiler that is connected to $20 \mathrm{~mm}$ (inner diameter) copper tubing which supplies a radiator and a buffer vessel. A condensing boiler is used as this is mandatory equipment for new buildings in most European Union member states [3]. Three pressure transducers monitor the system pressure. A fourth pressure transducer monitors the dissolved gas partial pressure in combination with a semi-permeable silicone membrane. Seven stainless steel sheathed $K$-type thermocouples are used to measure the fluid temperatures along the circuit.

The system fluid flow rate is monitored through an Electromag 500 Series electromagnetic flow meter. A National Instrument cDAQ-9172 chassis and relevant data modules receive all the signals from the transducers, thermocouples and electromagnetic flow meter. As tabulated in table 1, experiments were conducted to analyse the volumetric void fraction distribution across the vertical pipe sections. Therefore, sight glass VSG1 was used as illustrated in figure 1. The resultant void fraction was controlled through the dissolved gas concentration in the water, hence through the calculation of the resultant saturation ratio.

The saturation ratio, $\alpha(-)$, was calculated through the application of Eq. (1), as defined by Jones et al. [11]. This is the ratio of the actual dissolved gas concentration $C_{\text {gas }}\left(\mathrm{cm}^{3} \cdot \mathrm{L}^{-1}\right)$, present in the bulk fluid to the maximum concentration at saturation conditions $C_{\text {sat }}\left(\mathrm{cm}^{3} \cdot \mathrm{L}^{-1}\right)$. An analysis of the dissolved gas present in the closed loop system has shown that nitrogen is the dominant gas. This is a result of a limited oxidation following the system filling with fresh water. The oxidation process releases iron oxide and some hydrogen gas. The analysis of dissolved gases through the use of Orbisphere 3655 oxygen and Orbisphere 3654 hydrogen sensors resulted in very low concentrations of oxygen and hydrogen present in their dissolved form. In fact, both gases were present in concentrations of circa 9 PPB. Hence, nitrogen properties were used for the dissolved gas properties in the present study. The actual gas content, $C_{\mathrm{gas}}$, was calculated through the application of Eq. (2). The partial gas pressure, $p_{\mathrm{g}}(\mathrm{Pa})$, was calculated by subtracting the vapour pressure from the gas transducer reading as defined by Lubetkin and Blackwell [12]. $X^{\mathrm{T}}\left(\mathrm{cm}^{3} \cdot \mathrm{L}^{-1}\right.$ bar $^{-1}$ ), is the gas solubility factor.

$$
\begin{aligned}
\alpha & =\frac{C_{\text {gas }}}{C_{\text {sat }}} \\
C_{\text {gas }} & =p_{\mathrm{g}} X^{\mathrm{T}}
\end{aligned}
$$

A constant typical system pressure of 2.7 Bars (abs) was used for all experiments. This was set through the use of a nitrogen gas cylinder connected to a standard cylinder regulator. Also, a bulk fluid temperature of $80^{\circ} \mathrm{C}$ was maintained in the system flow line for all experimental runs. A constant heating load of $10 \mathrm{~kW}$ was maintained for all experimental runs.

The system flow rate or velocity is varied through the use of a ball valve on the supply line. The bulk fluid velocities in the system pipe work were set in the range of $0.19-0.52 \mathrm{~m}^{\cdot} \mathrm{s}^{-1}$. This is equal to a system volume flow rate ranging from $4.5-12.5 \mathrm{~L} \cdot \mathrm{min}^{-1}$. The saturation ratio or gas concentration in the system flow line was set through the variation in the nitrogen head contained in the upper part of the radiator with nitrogen gas.

As illustrated in figure 2, a square sight glass with internal dimensions of $20 \cdot 20 \mathrm{~mm}$ were used. A square section was designed to reduce the distortion as a result of viewing bubbles through a curved surface. As discussed by Prodanovic et al. [13], such distortions are due to light refraction. A Vision research Phantom V5 high speed camera connected to a PC was used to film and store the video clips.

The bubble distribution in the horizontal pipes was measured through the use of 6 focal planes at a depth of $1,4,8,12,16,19 \mathrm{~mm}$ across the vertical sight glass, VSG1 (figure 2). The main errors of this study originate from the limitations of the image analysis as discussed in Section 2.1 of the present study.

\begin{tabular}{|c|c|c|c|c|c|c|}
\hline $\begin{array}{l}\text { System } \\
\text { heating } \\
\text { load. } \\
(\mathbf{k W})\end{array}$ & $\begin{array}{l}\text { Internal } \\
\text { pipe } \\
\text { diameter } \\
(\mathbf{m m})\end{array}$ & $\begin{array}{l}\text { Bulk fluid velocity } \\
\text { in the system pipe } \\
\text { work } \\
\left(\mathrm{m}^{\cdot} \mathbf{s}^{-1}\right)\end{array}$ & $\begin{array}{c}\text { Bulk fluid } \\
\text { temperature at } \\
\text { system flow line } \\
\left({ }^{\circ} \mathrm{C}\right)\end{array}$ & $\begin{array}{c}\text { Maximum } \\
\text { saturation ratio } \\
\text { at boiler wall } \\
\text { conditions } \\
(-)\end{array}$ & $\begin{array}{l}\text { Heat flux } \\
\text { at the } \\
\text { boiler wall } \\
\left(\mathrm{kW} \cdot \mathrm{m}^{-2}\right)\end{array}$ & $\begin{array}{c}\text { Bulk fluid } \\
\text { velocity in } \\
\text { system pipe } \\
\text { work } \\
\left(\mathrm{m} \cdot \mathrm{s}^{-1}\right)\end{array}$ \\
\hline 10 & 20 & $0.19-0.52$ & 80 & 1.1 & 23 & $\begin{array}{c}0.19 ; 0.31 ; \\
0.42 ; 0.52\end{array}$ \\
\hline
\end{tabular}

Table 1. Experimental conditions. 


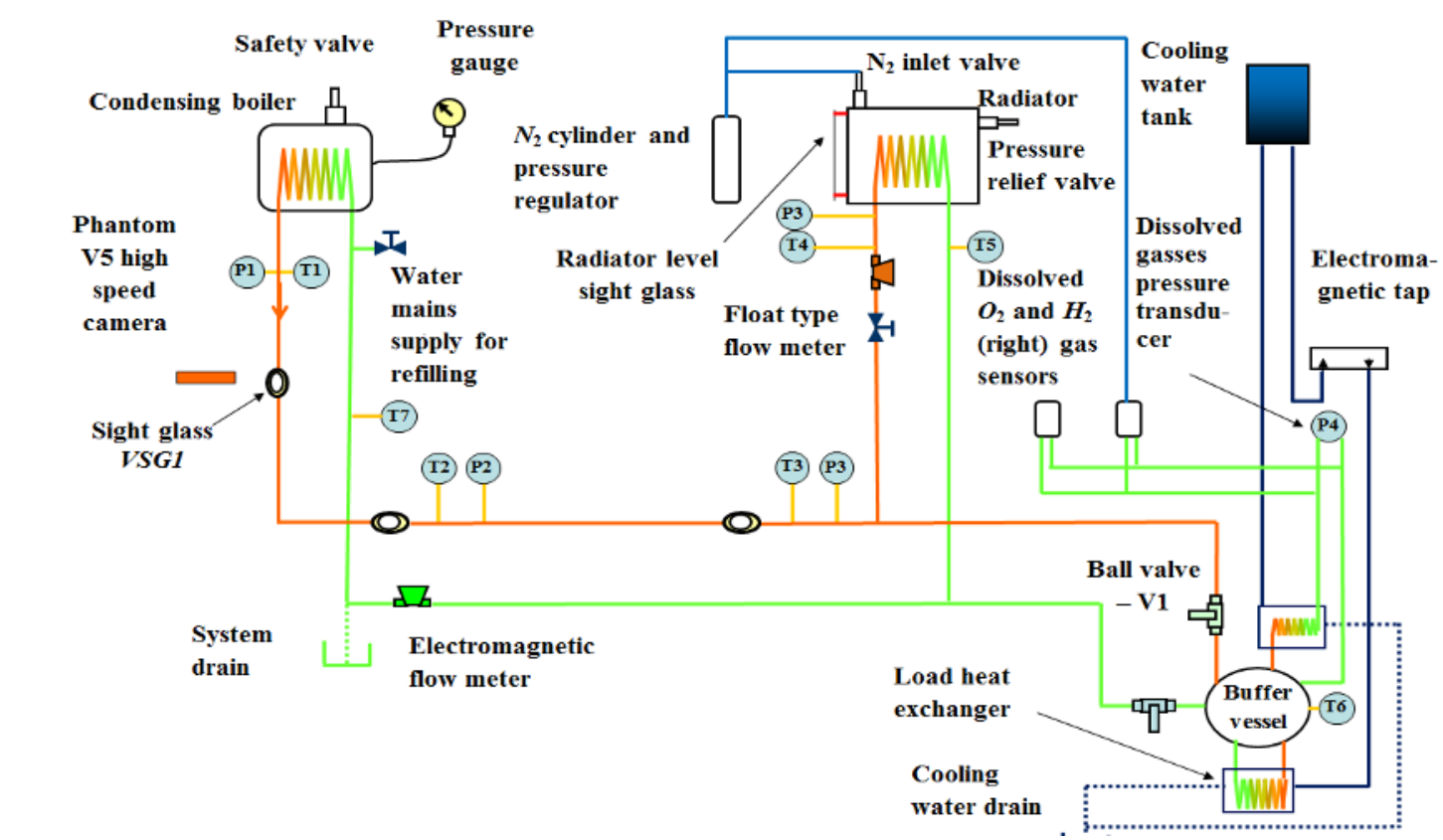

Fig. 1. Schematic diagram of the test rig.

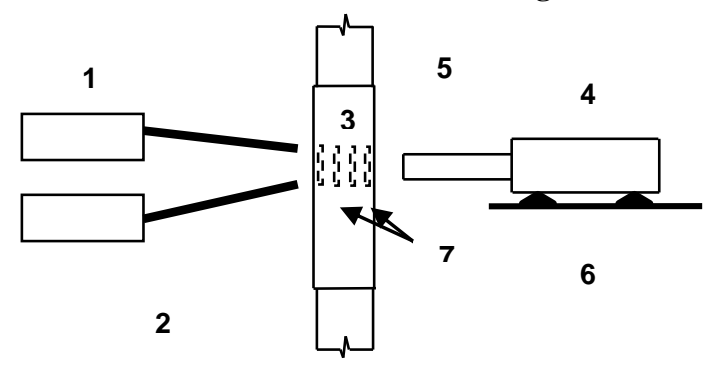

1. Light sources

2. Fibre op tic light guide

3. Square sight glass section

4. High speed camera
5. Microscope lens
6. PC wired to camera
7. Focal depth of $1.5 \mathrm{~mm}$
8. 5 focal planes

Fig. 2. Imaging equipment set-up for vertical sight glass, schematic (left), actual system set-up (right).

\subsection{Image analysis}

The video films were converted to image frames saved as tiff files using the Phantom Version 606 camera software. The resultant bubble densities and diameters were measured through the use of the image analysis software, Image-Pro Plus developed by Media Cybernetics. A macro was written enabling a series of images to be analysed for in focus bubble counts and diameters. The macro included the use of a Sobel filter to enable the distinction between in and out of focus bubbles. The Sobel filter plots the gradient of the intensity change between objects and their background through the extraction and enhancement of edges and contours. This is done by expressing intensity differences or gradients between neighbouring pixels as an intensity value.

Therefore, objects that are in focus have sharp edges with a high gradient change and consequently result in high intensity values, whereas out of focus objects do not display such a characteristic. The Sobel

filter was used as it is less sensitive to image noise when compared to other filtering techniques [14]. A typical analyzed image is illustrated in figure 3, where in focus bubbles are circled. Experimental uncertainties were calculated based on the method given by Coleman and Steel [14] and estimated as a mean absolute value of $19.6 \%$ for the volumetric void fraction $V F(-)$, calculated through the use of Eq. (3).

$$
V F=\frac{V_{\text {ba }}}{V_{\text {sv }}}
$$

Where $V_{\mathrm{ba}}\left(\mathrm{m}^{3}\right)$ is the average bubble volume per image and $V_{\mathrm{sv}}\left(\mathrm{m}^{3}\right)$ is the image sample volume calculated through the two-dimensional length and width of the recorded images as in figure 3, and the measured depth of field of $1.5 \mathrm{~mm}$.

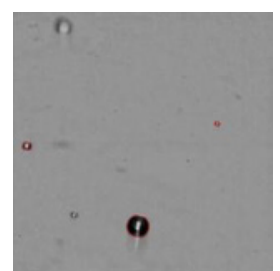

Fig. 3. Typical image (post processing with in focus bubble circled. 


\section{Experimental results and discussion}

This section presents the results for the bubble distribution at the boiler exit in a vertical downward bubbly two-phase flow. As illustrated in figures $4-8$, the results are presented through the measured volumetric void fractions across the pipe section, using sight glas VSG1 for the experiments as tabulated in table 1. This data is presented in relation to the position across the pipe section, represented through a dimensionless number $r_{\mathrm{p}} / R_{\mathrm{p}}$, (-), where a zero value signifies the pipe centre line. Hence, figure 4 summarizes the results whereas figures 58 provide the actual results for the tests conducted in the present study. After considering the errors due to the experimental uncertainty that amount to $\pm 19.6 \%$ of the readings values, the results, suggest that a quasi-flat volumetric void fraction profile is expected across the vertical pipe section for a downward two-phase bubbly flow.

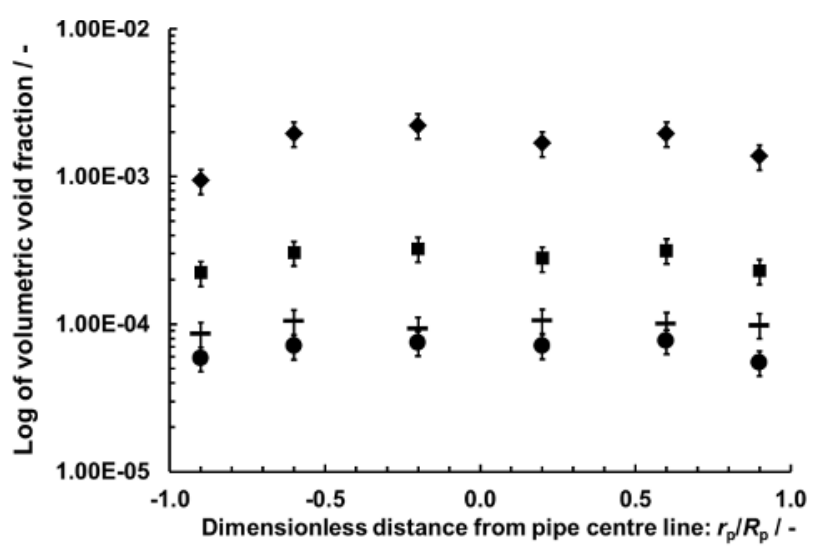

$\bullet \operatorname{Re}: 9.5 \mathrm{E}+3 /$ - $\square \operatorname{Re}: 15.9 \mathrm{E}+3 /$ - $\bullet \operatorname{Re}: 21.2 \mathrm{E}+3 /$ - - Re: $26.6 \mathrm{E}+3 /$ -

Fig. 4. Volumetric void fractions with dimensionless distance across the vertical pipe at the boiler exit with the bulk fluid Rey nolds number.

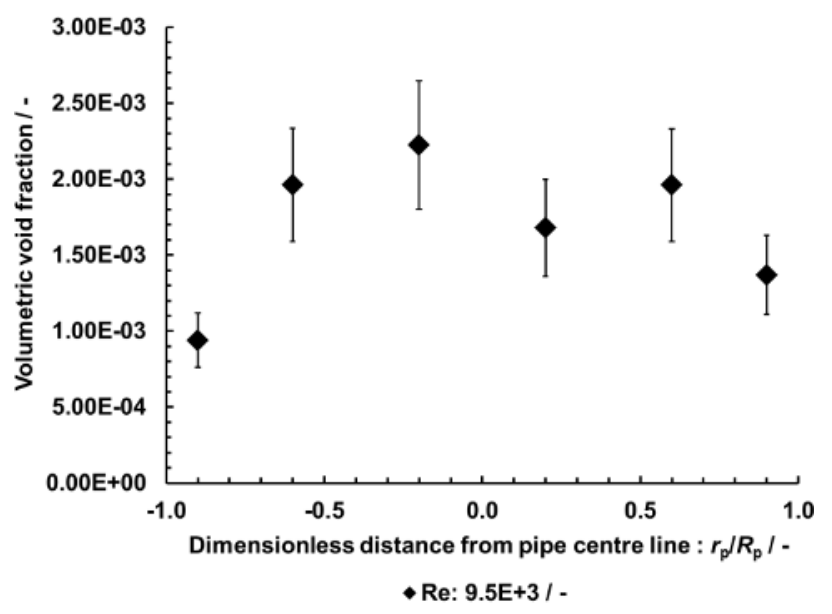

Fig. 5. Actual volumetric void fraction with dimensionless distance across the vertical pipe at the boiler exit flow line at a volume flow rate of $4.5 \mathrm{~L} \cdot \mathrm{min}^{-1}$.

In all four experiments, marginally lower volumetric void fractions and mean bubble diameters were measured at a distance of $1 \mathrm{~mm}\left(0.9 r_{\mathrm{p}} / R_{\mathrm{p}}\right)$ from the pipe wall. As illustrated in figure 9 , such a trend is more distinct at higher volumetric void fractions, where the lower system velocities were applied. Figure 4 suggests a trend where higher mean volumetric void fractions across the pipe section are present at lower bulk fluid velocities. This could be attributed to the large mean bubble diameters measured at low bulk fluid velocities [16]. However, at higher bulk fluid velocities, this trend is not evident and in fact, through the consideration of the experimental errors, there is no distinct difference in the volumetric void fraction measured at the two highest bulk fluid velocities resulting in a Reynolds number of $21.2 \mathrm{E}+3$ and 26.6E+3. This observation could be attributed to the higher bubble production rates with an increase in the bulk fluid velocity [17]. Therefore higher bubble counts compensate for any reduction in the bubble diameter with an increase in the bulk fluid velocity.

The studies done in bubbly vertical downward flow by Drew and Lahey [6], Wang et al. [7] Antal et al. [8] and Kashinsky and Randin [5] reported similar void fraction distributions as measured in the current study with a quasi-constant void fraction in the core region which drops abruptly to zero as the wall is approached.

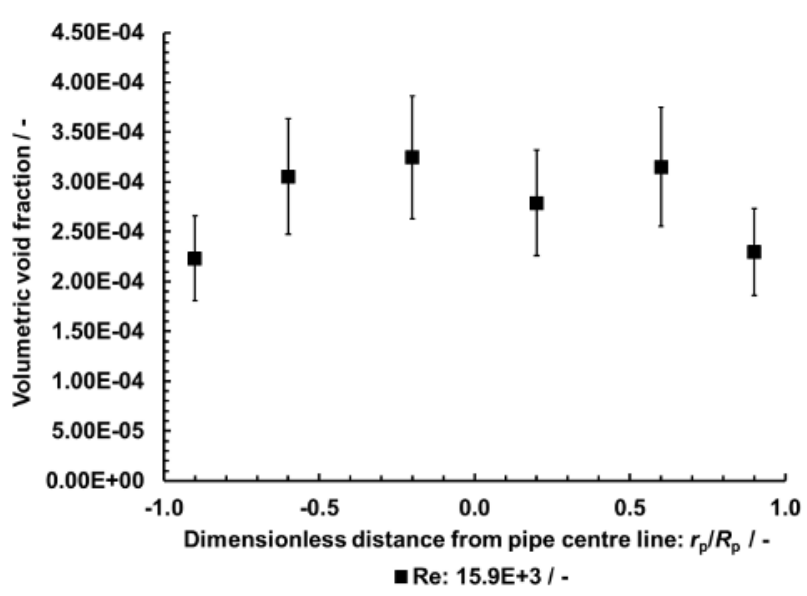

Fig. 6. Actual volumetric void fraction with dimensionless distance across the vertical pipe at the boiler exit flow line at a volume flow rate of $7.5 \mathrm{~L} \cdot \mathrm{min}^{-1}$.

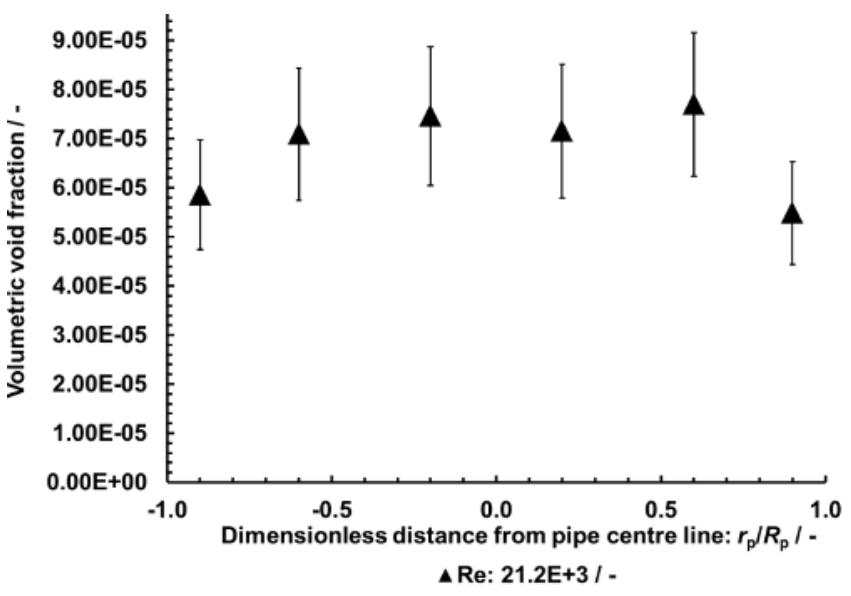

Fig. 7. Actual volumetric void fraction with dimensionless distance across the vertical pipe at the boiler exit flow line at a volume flow rate of $10 \mathrm{~L} \cdot \mathrm{min}^{-1}$. 


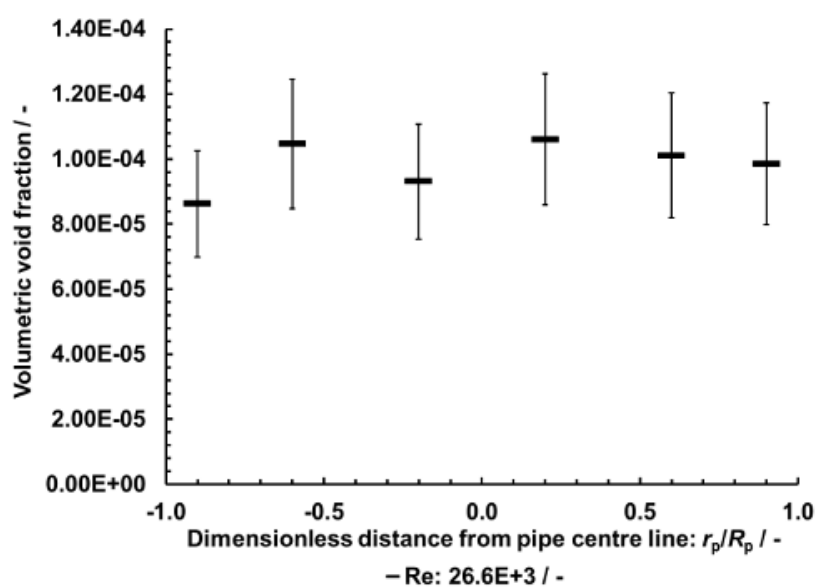

Fig. 8. Actual volumetric void fraction with dimensionless distance across the vertical pipe at the boiler exit flow line at a volume flow rate of $12.5 \mathrm{~L} \cdot \mathrm{min}^{-1}$.

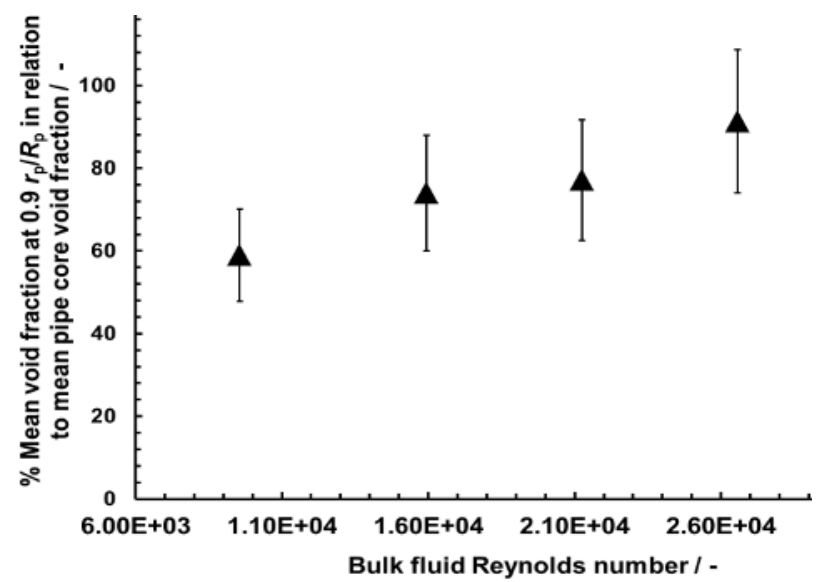

Fig. 9. Percentage mean volumetric void fraction at $0.9 r_{\mathrm{p}} / R_{\mathrm{p}}$ in relation to the mean void fraction measured in the pipe core with the bulk fluid Reynold's number.

Iskandrani and Kojasoy [9] also reported that, in twophase vertical bubbly flows, the presence of voids tends to flatten the liquid velocity profile, thus leading to a homogenous phase distribution across the vertical pipe section. More recently, Lu and Tryggvason [10] reported similar trends through direct numerical simulations.

Wang et al. [7] and Kashinsky and Randin [5] reported a drop in the void fraction at a mean distance of circa 0.9 of the pipe radius from the pipe centre line. The results presented in the current study should be considered in view of the relatively low void fractions present in the system. In fact, as illustrated in figure 5, maximum mean bubble diameters resulting in a mean volumetric void fraction of circa $2 \mathrm{E}-3$ were measured at the lowest bulk fluid Reynolds number of $9.5 \mathrm{E}+3$. Revankar and Ishii [18] and Liu [19] reported that in vertical two-phase pipe bubbly flow characterized with small bubble diameters and void fractions, uniform distributions are more likely across the pipe section in vertical fluid flow.

Kashinsky and Randin [5], reported that low bulk fluid velocities of $0.5 \mathrm{~m}^{\cdot} \mathrm{s}^{-1}$ in a pipe with an internal diameter of $42.3 \mathrm{~mm}$ resulted in more bubbles closer to the wall consequently improving the flatness of the void fraction distribution, with a drop in the void fraction starting at a distance of circa 0.95 from the pipe centre line. They reported that such an effect is more pronounced with the liquid velocity when compared to the bubble size or void fraction. This can be attributed to the reduced effect of liquid turbulence at lower bulk fluid velocities. Hence, such findings contrast to the results of the present study whereby larger void fractions at lower system velocities resulted in a reduction in the flatness of the void fraction distribution. Therefore, the present study suggests that with system fluid velocities less than 0.52 $\mathrm{m} . \mathrm{s}^{-1}$, equivalent to a Reynolds number of $26.5 \mathrm{E}+3$, the turbulence effects created by larger bubbles tend to have a greater effect on the void fraction distribution. Due to the increase in the bubble detachment diameter from the primary heat exchanger wall at lower fluid velocities, [16], the present study could not investigate void fraction distribution trends with velocity and bubble size independently.

Kashinsky and Randin [5] reported that in contrast to an upward flow, in downward bubbly flow, a velocity boundary layer close to the wall is expected as in the case of a single-phase flow. Hence, they referred to the conservation of the 'law-of-the-wall' in gas liquid bubbly flow as contributor to the drop in the void fraction in this area. They also reported that the resultant wall shear stress, or friction velocity, is an appropriate parameter for describing the near wall region in downward bubbly flows. Kashinsky and Randin [5] reported that the size of the gas bubbles produces a significant effect on the wall shear stress, thus increasing with bubble size, hence, in agreement with the results of the present study, the resultant change in the void fraction distribution with bubble size. They attributed this effect to the higher tubulisation of the flow by big bubbles for which both the size and the relative velocity are higher.

Žun [20] and Kashinsky and Randin [5] suggested that the main reason for the bubble migration in the flow away from the pipe wall is a transverse lift force acting on a bubble dependent on the phase relative velocity and the liquid velocity gradient. Furthermore, Antal et al. [8] related the void fraction distribution across the vertical pipe section with a wall repulsion force dependent on the bubble radius, distance from the wall and the phase relative velocity. Such a repulsion force is assumed to be equal for both down and upward flows. Hence, in a downward flow, both forces act in the same direction therefore pushing the bubbles away from the pipe wall at the region with the strongest velocity gradient, hence, the void fraction distributions as measured in the current study.

The bubble distribution trends for a vertical bubbly downward flow contrast with the expected void fraction distribution in upward vertical pipe flow. Serizawa et al. [21], Michiyoshi and Serizawa [22], Revankar and Ishii [18], Liu and Bankoff [23] and Hibiki et al. [24] reported that in contrast to the downward flow void fraction distribution, two-phase upward flow is expected to result in a peak void fraction close to the wall. Kashinsky and Randin [5] attributed this to the transverse lift force, as originally defined by Žun [20], acting on the bubble in an 
upward flow (with an opposite sign to that for a downward flow), thus leading to wall peaked void fraction distribution profiles across the pipe section. Hence, this contrasts to the 'coring' effect as defined by Drew and Lahey [6], whereby higher void fractions are expected at the pipe core in relation to the region close to the wall in two-phase bubbly downward flow characterized by high void fractions.

\section{Conclusions}

This paper has presented an experimental study on the typical phase distribution, quantified through the volumetric void fraction, in vertical two-phase bubbly flow as is typical in the flow line of a domestic central heating system due to gas super saturation conditions.

The present study has suggested a dependence of the phase distribution across the pipe depth on the degree of turbulence of the bulk fluid. Hence, at higher bulk fluid Reynolds numbers, a predominantly flat distribution is evident across the pipe section. A minimal reduction in the volumetric void fraction was measured close to the pipe wall at all bulk fluid velocities. At a lower bulk fluid degree of turbulence, the volumetric void fraction is significantly higher in the core of the pipe.

The importance of the present study lies with the fact that a comprehensive understanding of the phase distribution in the flow line pipes of central heating systems should lead to an optimised deaeration system, thereby improving the overall system performance, hence reducing the extensive carbon footprint of such systems. An exact quantification of the benefits of an enhanced deaeration on the system performance cannot be quantified precisely as minimal relevant data is available in the open literature. However, a reduction in the overall bubble count in the system pipework is known to reduce the susceptibility of such systems to problems such as radiator cold spots, excessive noise, pipework vibration and cavitation corrosion.

Further studies should be undertaken as an investigation of the bubble distribution in horizontal pipes. Such work could also be extended to the effects of pipe work bends on the resultant distribution in straight horizontal pipes.

\section{Acknowledgments}

The authors would like to thank the Engineering and Physical Science Research Council and Spirotech b.v. for supporting this research work.

\section{References}

1. W. Gerrard, Solubility of gases and liquids, Plenum Press, New York (1976)

2. C.L. Young, R. Battino, H.L., Clever, The solubility of gases and liquids - introductory information, Nitrogen and Air, solubility data series, Pergamon Press, Oxford (1982)
3. Office of the Deputy Prime Minister, The Building Regulations, Conservation of Fuel and Power - Part L1A, Office of the Deputy Prime Minister (2006)

4. R.H.S. Winterton, J.S. Munaweera, J. Chemical Engineering and Processing, 40, 437-447 (2001)

5. O.N. Kashinsky, V.V. Randin, Int. J. Multiphase Flow, 25, 109-138 (1999)

6. D.A. Drew, R.T. Lahey, J. Fluid Mech., 117, 91-106 (1982).

7. S.K. Wang, S.J. Lee, O.C. Jones Jr, R.T. Lahey Jr, Int. J. Multiphase Flow, 13 (3), 327-343 (1987)

8. S.P. Antal, R.T. Lahey Jr., J.E. Flaherty, Int. J. Multiphase Flow, 17 (5), pp. 635-652 (1991)

9. A. Iskandrani, G. Kojasoy, J. Nuclear Engineering and Design, 204, 117-128 (2001)

10. J. Lu, G. Tryggvason, J. Chemical Engineering Science, 62, 3008-3018 (2007)

11. S.F. Jones, G.M. Evans, K.P. Galvin, J. Advances in Colloid and Interface Science, 80, 27-50 (1999)

12. S. Lubetkin, M. Blackwell, 1988, J. Colloid and Interface Science, 26, 610-615 (1988)

13. V. Pradanovic, D. Fraser, M. Salcudean, Int. J. Multiphase Flow, 28, 1-19 (2001)

14. Image-Pro, Image-Pro Plus Start-Up Guide, MediaCybernetics (2010)

15. H.W. Coleman, W.G. Steele, 1999, Experimentation and uncertainty analysis for engineers, $2^{\text {nd }}$ ed., New York, John Wiley\& Sons Inc. (1999)

16. A.M. Fsadni, Y.T. Ge, A.G. Lamers, Applied Thermal Engineering, 31, (14-15), 2808-2818 (2011)

17. A.M. Fsadni, Y.T. Ge, A.G. Lamers, Applied Thermal Engineering, 45, 24-32 (2012)

18. S.T. Revankar, M. Ishii, Int. J. Heat and Mass Transfer, 35 (4), 913-925 (1992)

19. T.J. Liu, Int. J. Heat and Mass Transfer, 19, 99$113(1993)$

20. I. Žun, Int. J. Multiphase Flow, 6, 583-588 (1980)

21. A. Serizawa, I. Kataoka, I. Michiyoshi, Int. J. Multiphase Flow, 2, 235-246 (1987)

22. I. Michiyoshi, A. Serizawa, Turbulence in twophase bubbly flow, Nuclear Engineering and Design, 95, 253-267 (1986)

23. T.J. Liu, S.G. Bankoff, Int. J. Heat and Mass Transfer, 36 (4), 1061-1072 (1993)

24. T. Hibiki, R. Situ, Y. Mi, M. Ishii, Int. J. Heat and Mass Transfer, 46, 1479-1496 (2003) 